\title{
How Do You Know When You Have Interoperability?
}

\author{
William Ed Hammond ${ }^{1,2 *}$ \\ ${ }^{1}$ Duke Center for Health Informatics, Duke Medicine, Durham, North Carolina, USA \\ ${ }^{2}$ Duke Clinical and Translational Science Institute, Duke University, Durham, North Carolina, USA
}

\begin{abstract}
This paper first looks at the several definitions of the word interoperability. Next, the paper addresses several of the distinct components that are factors in accomplishing interoperability: semantic interoperability, functional interoperability, stakeholder interoperability, consumer interoperability, business interoperability, privacy and
\end{abstract}

Correspondence to:

W. Ed Hammond PhD, FACMI, FAIMBE, FIMIA, FHL7

Duke Center for Health Informatics, NC 27705, USA.

E-mail: william.hammond@duke.edu security interoperability, and international interoperability. This paper discusses each of these components in terms of their current state relating to interoperability and suggests what changes might be made to achieve interoperability.

\section{Keywords}

Interoperability; Health data standards; Terminologies; EHRs; PHRs; Business models; Consumers

\section{EJBI 2018; 14(3):13-20}

Received: May 15, 2018

Accepted: May 22, 2018

Published: July 06, 2018

\section{Introduction}

Interoperability has been the Holy Grail of Informatics for many decades. Its definitions have been diffuse, and its obtainability has been seemingly impossible. It was an impetus for the creation of Standards Developing Organizations (SDO); the SDOs used the term "plug and play" although we have never reached that point. In the United States, the Office of the National Coordinator (ONC) has made interoperability a focus of its initiatives. "How do you know when you have interoperability?" is almost an unanswerable question; or else it may have innumerable answers. Part of the problem is in understanding the boundaries of the term. If I am able to exchange and understand the exchange of a single data element and that is all I care about, is that an interoperable system? If I can exchange and understand a complete patient summary, is that interoperability? If I can map from a local vocabulary to a common master set of terminologies, does that constitute interoperability?

This paper will not provide an answer to the question in its title. Instead, the paper will provide a fresh look at the many issues of interoperability from both an overall perspective plus a critical analysis of several specific components of interoperability. I will discuss new perspectives of traditional views of interoperability and suggest alternate approaches. The paper proposes that interoperability requires more than the transfer data and even more than the understanding the meaning of that data. Interoperability includes the appropriate use of that data; dealing with privacy and security; dealing with regulations; dealing with quality of the data and with trust; dealing with authentication and authorization; dealing with governance; and dealing with the many stakeholders who have a vested interested in the data and its use.

\section{Definitions of Interoperability}

The earliest views of interoperability came from IEEE in 1990, defining interoperability as "the ability of two or more systems or components to exchange information and to use the information that has been exchanged" [1]. The ability to exchange information is referred to as functional interoperability, and the ability to use that information is called semantic interoperability. This definition became the driving force for the development of data exchange standards and standard terminologies. My observation is that the word information should be replaced by the word data. That distinction between the word data and information is increasingly important.

Health Level 7 International ${ }^{\circ}$ uses the IEEE definition of interoperability but adds more detail [2]:

- "Functional" interoperability is the capability to reliably exchange information without error

- "Semantic" interoperability is the ability to interpret, and, therefore, to make effective use of the information so exchanged.

The recognition of the effective use of the information is an important addition, although no detail is provided about how to do that. 
The HIMSS definition [3] builds further on the concepts within the HL7 definition: interoperability means the ability of health information systems to work together within and across organizational boundaries in order to advance the effective delivery of healthcare for individuals and communities. HIMSS defines three levels of health information technology interoperability:

- Foundational - allows data exchange from one information technology system to be received by another and does not require the ability for the receiving information technology system to interpret the data.

- Structural - defines the structure or format of data exchange (i.e., the message format standards) where there is uniform movement of healthcare data from one system to another such that the clinical or operational purpose and meaning of the data is preserved and unaltered. Structural interoperability defines the syntax of the data exchange. It ensures that data exchanges between information technology systems can be interpreted at the data field level.

- Semantic - the ability of two or more systems or elements to exchange information and to use the information that has been exchanged. Semantic interoperability takes advantage of both the structuring of the data exchange and the codification of the data including vocabulary so that the receiving information technology systems can interpret the data. This level of interoperability supports the electronic exchange of patient summary information among caregivers and other authorized parties via potentially disparate electronic health record (EHR) systems and other systems to improve quality, safety, efficiency, and efficacy of healthcare delivery [4].

Dr. Bernd Blobel provides an outstanding discussion of interoperability and its component parts in his article "Standardization for Mastering Healthcare Transformation“ [5].
He introduces an Interoperability Reference Architecture Model that provides direct methods for dealing with the different instances of interoperability and includes a discussion of challengres and solutions. In a related paper, Dr. Blobel further discusses challenges, standards, and solutions for EHR systems interoperability [6]. Kevin Heubusch also provides an interesting discussion of Interoperability from the perspective of ONC [7].

From these varied definitions from key, relevant organizations, interoperability is defined mainly from the technical aspects. In truth, interoperability involves a much larger scope of interests. My working definition of interoperability is the ability to share data whose meaning is unambiguously clear, its context understand, and it can be used for whatever purpose - and - the receiver is not previously known to the sender; i.e., an open-loop process. Even so, this is a limited definition that will be expanded below. Figure 1 shows many addition units that have concerns, interests and influence on interoperability.

\section{Semantic Interoperability}

\subsection{ICD}

From the moment two persons tried to communicate, semantic interoperability became important. Within the health care industry, semantic communication became important, first between individuals with the same clinical units, then within the same institutions, between clinical departments. With the current interests in data sharing, semantic communications have become increasingly important.

The first international classification was for the list of causes of death, adopted by the International Statistical

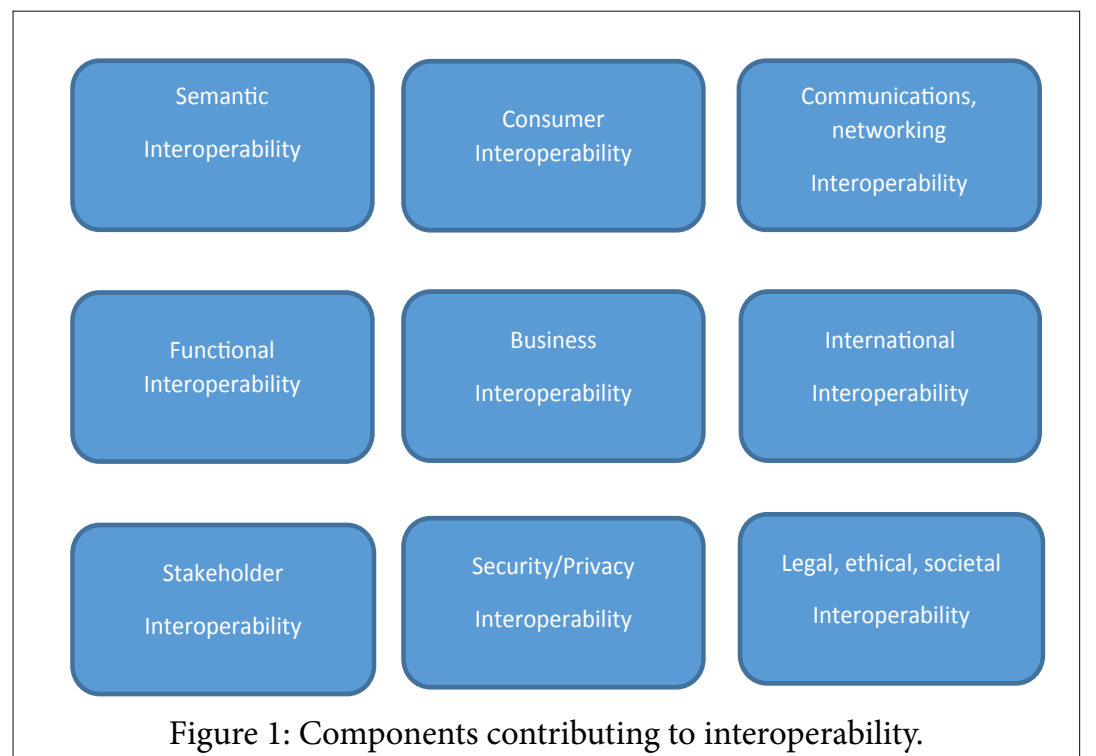


Institute in 1893. WHO was entrusted with the ICD at its creation in 1948 and published the 6th version, ICD-6, that incorporated morbidity for the first time. ICD-10 was endorsed in May 1990 by the Forty-third World Health Assembly and used by more than 100 countries around the world. ICD-10 [8] uses include monitoring of the incidence and prevalence of diseases, observing reimbursements and resource allocation trends, and keeping track of safety and quality guidelines. They also include the counting of deaths as well as diseases, injuries, symptoms, reasons for encounter, factors that influence health status, and external causes of disease.

Although ICD serves an important purpose, it does not address the requirements for semantic interoperability.

\subsection{SNOMED-CT}

SNOMED CT traces its history back to 1965 with the publication of the Systematized Nomenclature of Pathology (SNOP), published by the College of American Pathologists (CAP) to describe morphology and anatomy. In 1975, under the leadership of Dr. Roger Cote, CAP expanded SNOP to create the Systematized Nomenclature of Medicine (SNOMED). The most widely adopted version of SNOMED was SNOMED II (1979), followed by an expanded revision called SNOMED International (1993). CAP and Kaiser Permanente developed a logic-based version, SNOMED RT, in 2000. The Read Codes developed by Dr. James Reed in the United Kingdom were merged into SNOMED - Clinical Terms in the 1980s. The current version of SNOMEDCT has evolved from that set [9].

Although SNOMED has an increased use in the US and other countries, it can be used only by countries that have paid the licensing fee. Further, the terminology itself does not cover all clinical terminology and is complicated by pre and post coordination. The words of SNOMED are not the words used by professionals to define and describe clinical communication.

\subsection{Logical Observation Identifiers Names and Codes (LOINC)}

LOINC [10] is widely used internationally for representing clinical results such as laboratory tests, clinical observations, outcomes management and research. LOINC has two main parts: laboratory LOINC and clinical LOINC. Clinical LOINC contains a subdomain of Document Ontology which captures types of clinical reports and documents. Although LOINC comes close to meeting the requirements for semantic interoperability, the multiple names for the same tests creates major issues in exchanging and understanding the data that is represented. A solution for laboratory and other tests would be the assigning a LOINC code when the test was performed by the performing unit based on an agreed standard set throughout the industry.

\subsection{Drug Coding}

The coding of pharmaceutical products is perhaps one of the most challenging issues in semantic interoperability. In the US, RxNorm [11], developed by the National Library of Medicine, is coming into increased use. RxNorm incorporates data and knowledge from several sources.

A problem with drug coding is that each country has its own formulary and therefore its own drug terminology. Patients typically have drugs prescribed by different providers and at different locations. Bringing these medications together to create a prescription history is critical in the care of a patient. Although medication profiles are one of the most important aspect of a patient's history, it is the most difficult to achieve.

\subsection{Addressing Semantic Interoperability}

There are a number of reasons why semantic interoperability has evaded us for decades. First, there are many terminology sets currently in use today, with local terminologies being the most common. We seek a solution from common controlled terminologies that are incomplete and do not match the required clinical representation. Most of the terminology coding are influenced by reimbursement and do not represent the terms and the granularity required by direct clinical care. Cancer research is frustrated by the lack of a common vocabulary to support research for example in dealing with tumors. Then, there is confusion about the relationship among vocabularies, terminologies, classifications, nomenclature, ontologies, data models, and data elements. What role does each play in communications and semantic interoperability?

We are trying to solve the problem with what currently exists, rather than understanding what semantic interoperability is about. It is about communication, and we need to communicate at the level of need and understanding. Today's problem with semantic interoperability is that our first approach is mapping between terminologies to a common data model. Mapping inherently loses information. If the mapping does not, then why have two terminologies. Secondly, the cost is high because most terminologies are changing, and the mappings are out of synchronization.

There are a number of efforts to create a common data model as an approach to semantic interoperability. Unfortunately, there are enough common data models in health care so that they become uncommon. Examples include Sentinel, OMOP, i2b2, PCORNet, HL7, NLM VSEC, CDISC, CIMI, CIIC, and many others.

A single, international, data set of data elements offers the best solution in my opinion. The data element can be 
knowledge metamodel with a rich set of attributes that can define all knowledge associated with the data element. Figure 2 illustrates typical attributes that might exist to contain the knowledge relating to the data element. Further, a process must be defined and implemented that would permit additional data elements to be added with a vetting process. Such a system would eliminate the need for locally-defined data elements that lose semantic interoperability. With such a common set of data elements defined, every local site should be required to adopt the set within a short period of time. Other authors have addressed this problem of data modeling and its contributions to interoperability [12].

\subsection{Data Collection}

The intangible component of semantic interoperability is data quality and consequently trusts. Data collection, then, is a necessary consideration for semantic interoperability. First, whenever possible, data collection must be automated. Wearable sensors are a step in that direction and increasingly can measure key parameters related to a person's real time health. If we can determine medical errors after they occur, we need to use the same algorithms in real time to prevent the error from occurring. If we have algorithms that will clean the data, we need to employ those same algorithms as part of the data collection to establish data quality.

\subsection{New Data Types}

The types of data that have value in clinical decision making has expanded significantly in recent years. In an Institute of Medicine (now the National Academy of Medicine) 2002 publication, J.
McGinnis [13] claims that clinical data contributes only $10 \%$ information to a person's health index. The other data types are behavioral (40\%); genomic (30\%); social/economic (15\%); and environmental (5\%). We now need to create semantic interoperability to include these data types, and we need to define ways to capture and include this data in EHRs.

\section{Functional Interoperability}

\subsection{HL7 International Standards}

There are a number of standards developing organizations (SDOs) that have created data exchange standards. HL7, created in 1987, is a leader in the field and has created a continuing progression of standards. The first HL7 standard for the exchange of data is known as v2.n, where the current version is v2.8. Over $95 \%$ of hospitals and clinics use some version of HL7 v2 today. A second HL7 standard in wide use today is based on the v3 model-based standard and is known as the Clinical Document Architecture (CDA). CDA defines the structure of certain medical records, such as discharge summaries and progress notes, as a way to better exchange this information between providers and patients. An Implementation Guide based on this standard is in wide use to transfer the Patient Summary. This standard is known as the Continuity of Care Document (CCD).

The most recent HL7 data transfer standard is called the Fast Healthcare Interoperability Resource (FHIR') standard [14]. It is a web-based standard and uses the REpresentational State Transfer (REST). REST is an architectural style that defines a set of constraints and properties based on HTTP.

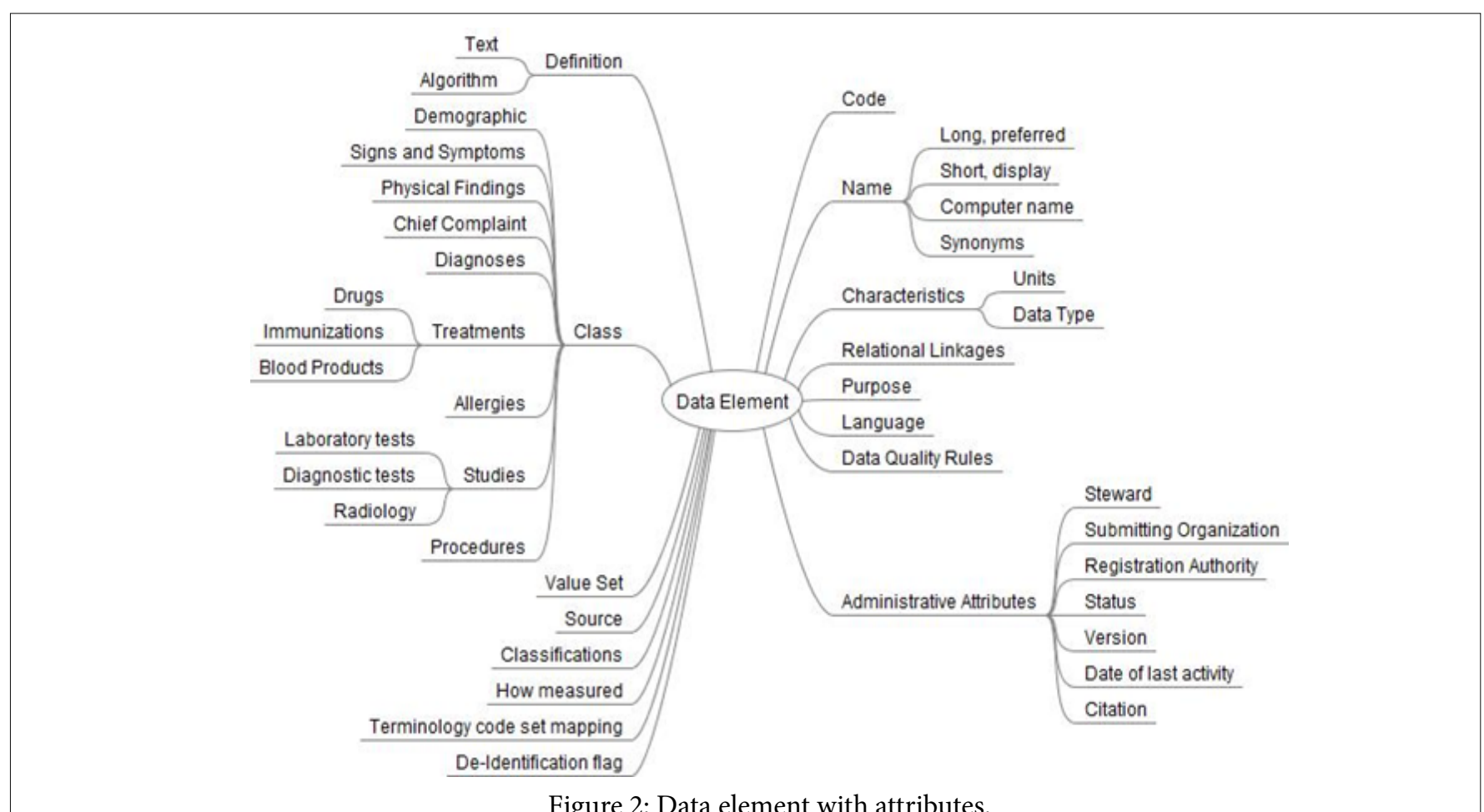


Web Services that conform to the REST architectural style, or RESTful web services, provide interoperability between computer systems on the Internet. Facebook, Google, and others use this standard. RESTful systems typically communicate over HTTP verbs (Create/Post, Read/Get, Update, and Delete). FHIR provides interoperability between computer systems over the Internet.

FHIR is built on logical, related compound structures called RESOURCES. Resources consist of small logically discrete units of exchange with defined behavior and meaning. Resources have a known identity and location identified by a Universal Resource Identifier (URI). All exchangeable content is defined as a Resource. There are over 150 different Resources that are intended to cover $80 \%$ of healthcare. Examples include Patient, Practitioner, Family History, Care Plan, and Allergy Intolerance. Resources are defined using XML, JSON, or RDF. The core Resources reside in a repository open and free to use for all.

Resources are combined into groups called PROFILES to identify packages of data to address clinical and administrative needs. Parties exchanging data define the specific way they want to use Resources and their relations using Profiles. FHIR is service driven. Profiles define what a particular application needs to communicate based on Resources and Extensions (data elements, self-defined, that are not part of the core set). You only send data that is required for specific purposes. Profiles are used to constrain Resources - that is to define specifically what data is to be sent. Examples of Profiles are for referral of a patient; for populating registries; adverse event reporting; ordering a medication; and providing data to a clinical decision support algorithm such as a risk assessment calculation.

The FHIR standard has the potential to transfer any and all types of data. If FHIR Resources are tightly bound to a global master set of data elements, then functional interoperability becomes more achievable. The remaining barrier to interoperability is extensions. Extensions seem necessary to accommodate the transfer of data beyond the standardized core data elements. Unfortunately, that freedom opens the door to creating innumerable, uncontrolled, and potentially duplicative exchanges. The problem is further complicated by the $80 / 20$ decision. FHIR core resources will address only $80 \%$ of the data more commonly required and the remaining $20 \%$ will be accommodated by extensions. I propose a better solution would be to define a process in which resources and data elements would be submitted to HL7 to become part of the core set. These additional submissions would be properly vetted and move into the normative standard. As we look into the requirements of the new data types suggested above, and as we consider new requirements such as population health, many of the data elements have not been included in the standard set.

\subsection{SMART $^{\circledR}$ on FHIR}

SMART is an open standards-based technology [15] that enables developers to create apps that seamlessly and securely run across the healthcare system without requiring specific knowledge about each system. Many clinical apps have been built on this platform and are available through a publically accessible app gallery. SMART on FHIR is a set of open specifications that builds on FHIR API and Resource definitions. FHIR provides the core data models and SMART defines the profiles that carry out the functions of the app. Additionally, SMART uses an authorization model for apps based on the OAuth [16] standard. OAuth permits patients and providers control of their data.

\subsection{CDS Hooks}

CDS Hooks [17] represents the third type of standard that is important to the use component of functional interoperability. Examination of the causes of medical errors, inconsistencies in care, missed opportunities, and other similar events is the inherent fallacies of humans to perform tasks consistently. CDS Hooks provides a way in which the hooks are inserted into the data flow to trigger external events such as clinical decision support algorithms. Josh Mandel, one of the developers of CDS Hooks, discusses in more detail this functionality and gives a number of excellent examples [18].

\subsection{Other Comments on Functional Interopera- bility}

The above section focuses primarily on HL7 standards. Other SDOs, including IHE, ISO, DICOM, IEEE, and others, contribute to the set of standards that have value in enabling functional interoperability. The good news is that most of these other SDOs are working with HL7 and are using FHIR in their standards. The new Gemini Project between HL7 and IHE should more tightly bind those collaborations. For example, IHE is creating profiles based on FHIR. With the new focus on imaging standards, particularly 3D images, close cooperation between SDOs become critically important. Obviously, FHIR resources can be defined to encapsulate these objects.

\section{Stackholder Interoperability}

A lesson learned from years of experience is that unless critical stakeholders are engaged and supportive, no new initiatives will change the current system. In its simplest way, I suggest healthcare is a matter of defining the problem, administering the appropriate medication, and monitoring the result. That works only if the payers will pay for it.

Stakeholders are key to interoperability. It is important to know who plans the strategy and who makes the decision about healthcare infrastructure. That may vary among countries - which then may influences differences that must exist in healthcare IT systems. In the US, I think the most influential stakeholders are payers and pharma. Next are the government, specifically FDA, CMS, CDC, and ONC. 
Laboratory vendors play a secondary role because that data is critical to defining the problem and measuring the effectiveness of the treatment. Consumers are becoming increasingly important but lack an organization to influence. Beyond that come other government agencies, researchers, health IT venders including EHR vendors, academic medical centers, and providers of care. An important observation is that to maximize the use of technology for better clinical interoperability, the reimbursement process must become secondary to clinical care process.

\section{Consumer Interoperability}

Ultimately, the purpose of the health care system is the health and well-being of the person. Until recently, the consumer has been the silent and invisible partner of healthcare. We could not have access to our health data, our preferences were never asked, and we were dominated by the healthcare system. That now is a changing world.

We now recognize the value of aggregating a patient's data into a single record - the Patient-Centric EHR. A simple challenge that has been difficult to achieve is a person's medication history. Patients typically will have medications including immunizations administered in more than one site. In the absence of a universal person identifier, there is a significant error rate in identifying a person who has data in several systems. We have created algorithms to identify persons but the error rates are still prohibitive to aggregate patient data with the required accuracy. Data frequently are entered into the wrong person's record. Clinical trials across multiple systems are biased by duplication of records. Most countries do have a universal person identifier; the US does not.

The healthcare process is supported from an IT perspective by EMR or EHR systems, and from a person perspective by a Personal Health Record (PHR). Typically, all functionality related to capturing, analyzing, presenting, and using that data is contained with the EHR. Unfortunately, much data created from the patient is not contained within the EHR. Caregivers and research complain of the difficulty of getting access to the data for any external purpose, such as used in a CDS algorithm or populating a registry. Furthermore, users are limited to the functionalities provided by the vender. Most of the dominant EHR systems are more than 40 years old. Technology has progressed well beyond the technology that exists in these commercial systems. To increase users' interoperability, I propose we create a new approach. We replace the current EHR with a Digital Data System (DDS) whose sole purpose is the intake, storage, and output of data. No functionality other than data in and data out exists. Functionality exists outside the DDS and permits a competitive environment among vendors, incorporates new and changing technology as well as new requirements, and permits specialization among specialties. Furthermore, such an approach would require the use of a common set of data elements for partication in the DDS. To the rest of the world, the DDS could function as a black box, without worrying about its internal workings, only about its performance.

Patients in most countries now have access to their health data either by a full record download or through a browser to view data. Rather than have a PHR, programs would exist to retrieve data from the DDS as required by the patient. It seems that a primary reason for providing access of a patient to their health data is to control that data. I think that misses the point. I want access to that data to better understand my health and how to manage it. First, my institutional EHR system contains data only when I am sick or when I visit for my annual exam. In my case, that data is a set of lab tests, vital signs, a problem list, and some demographic data. I look at it to see what is within normal limits. If a test has results outside number limits, I educate myself about what I can do to bring it back within normal limits. That might be a behavior change, or it might be a visit to the doctor. In any case, it is only a snapshot of my health status. What I want to do is to monitor my status as I live my life with my daily activities. Technology now permits that to happen with technologies including wearable sensors with real time data collection. What I want is a system that analyzes these data streams and makes decisions about my current state. I want a system that puts my data back into my healthcare system and alerts a provider when appropriate.

\section{Bussiness Interoperability}

Business aspects of the healthcare system dominate all other components of the system. The finances of an institution appropriately drive the Chief Financial Officer. Decisions about with which data may be shared are influenced by the CFO. Policies are driven by the business concerns. Most of the analytics done on health data are performed for business purposes. The balance between financial and healthcare delivery are a critical decision for an institution.

\subsection{Governance Interoperability}

As organizations share more and more of data and resources, governance becomes an important factor in interoperability. Governance rules are critically important among groups whether health care organizations, SDOs, affiliations, collaborators, or patients. Governance must establish a set of rules precisely defining ownership, flow, what can be done, and other activities.

\subsection{Regulatory Interoperability}

The purpose of regulations is the safety of the person. Regulations are critical for patient safety. Today we live in a rapidly changing world. Regulations should be reviewed frequently to be sure they are neither too stringent nor too 
loose. Interoperability does depend on matching patients across multiple databases. Regulations in the US currently make that impossible to do without an unacceptable error.

\section{Private and Security Interoperability}

Addressing privacy requirements is one of the challenging problems in interoperability. Both the Health Insurance Portability and Accountability Act (HIPAA) [19], enacted in 1996, and the newly enacted General Data Protection Regulation (GDPR) [20] define rather strict rules that regulate the exchange of data. Both sets of regulations control what data can be exchanged, and particularly identified data. The rules are focused on the privacy of an individual. Patient consent is generally in the use of identified and sometimes de-identified data. I suggest that for interoperability, a backwards linkage to a patient should be possible. If as a result of a clinical trial, new knowledge is generated that would have a positive effect on a patient that linkback would be important for better outcomes. Combining patient data of all types to create big data is critical for clinical research and the discovery of new data.

\section{International Interoperability}

Interoperability across a country's borders is perhaps the most challenging component to solve. First is the language barrier. Not all concepts translate across languages the same. In some cases, the translation is a physical description of the word. A single language used across all of health care is a possible solution, with demographics such as name and address be expressed in the native language. The obvious choice of such a language would be English, since most countries now teach English throughout all grade and high school levels. Another challenge is the accommodation of culture into the EHR and into treatment. Finally international interoperability accommodation for national drug formularies require continuous mapping from each country's formulary to a master set back to the second country. A global master formulary would save millions of dollars, but that is unlikely to ever happen.

\section{Conclusion}

It should be clear that I have not answered the question of how do you know when you have interoperability. However, the question does provide an opportunity to increase awareness of all the factors that have some influence on interoperability. From a technical perspective, we have examined more closely semantic functional interoperability. For semantic interoperability, the problem seems to be that too many groups are trying to solve the problems resulting in a non-solution. Controlled terminologies have increasing widespread use and have some intellectual value. Reimbursement, at least in the US, is likely to continue to be the dominating factor in the terminologies that are in use. The gap between what are clinically required vocabularies and the coding for reimbursement will likely be unchanged. We have chosen to use mapping among terminologies to avoid a valued solution. We do workarounds rather than solve difficult problems. Functional interoperability seems to be moving toward a workable solution with HL7's FHIR, SMART, and CDS Hooks. The agreements among the several SDOs will further contribute to functional interoperability.

Stakeholder interoperability is moving towards interoperability in that competitive groups are establishing trust and are defining what they require from the systems. By working together, these groups will define their requirements and share common solutions that can be provided from the SDOs working together.

Consumer interoperability continues to grow in importance and influence. What consumers want will push standardization in mobile devices, in wearable sensors, and in other Internet of Things. There seems to be less pushback on a universal unique personal identifier, and I think it is likely that we will finally adopt a UPID within a couple of years.

New technologies, new healthcare delivery models such as value based care, new policies and other change driving events will demand new business models. Although financial considerations will remain as the strong driving force, business models will better relate to where the other components of interoperability are going. Security will remain a major interest with new steps to contain hacking. Privacy will change to better address what is required for better health for individuals and research to provide new knowledge. As clinical research moves to pragmatic clinical trials using EHR data, the consistency and quality of EHR data will improve. Regulations also will address the use of AI and robotics as part of healthcare delivery. Governance is an essential component of data sharing among institutions.

The prospect for the future looks bright. Quality of life as well as length of life will improve for most of society. Population health will increase the focus on communities and on disparities in those communities. Developing countries will benefit from new technologies and new models of care. Interoperability is good.

\section{Acknowledgement}

Research reported in this publication was supported by the National Center For Advancing Translational Sciences of the National Institutes of Health under Award Number UL1TR002553. The content is solely the responsibility of the author and does not necessarily represent the official views of the National Institutes of Health.

\section{References}

[1] Geraci A, Katki F, McMonegal L, Meyer B, Lane J, Wilson P, et al. IEEE Standard Computer Dictionary: A 
Compilation of IEEE Standard Computer Glossaries. New [10] en.wikipedia.org/wiki/LOINC

York: IEEE Press; 1990.

[11] www.nlm.nih.gov/research/umls/rxnorm

[2] www.HL7.org

[3] HIMSS Dictionary of Healthcare Information Technology Terms, Acronyms and Organizations, 2nd Edition, 2010, Appendix B, p190, original source: Wikipedia.

[4] HIMSS Dictionary of Healthcare Information Technology Terms, Acronyms and Organizations, 2nd Edition, 2010, Appendix B, p190, original source: HIMSS Electronic Health Record Association.

[12] Blobel B and Oemig F. Solving the Modeling Dilemma as a Foundation for Interoperability. Eur J Biomed Inform. 2018; 14(3): 03-12.

[13] McGinnis JM, Williams-Russo P, Knickman JR. The case for more active policy attention to health promotion. Health Aff. (Millwood) 2002; 21: 78-93.

[14] www.hl7.org/fhir/

[15] smarthealthit.org

[5] Blobel B. Standardization for mastering healthcare transformations-challenges and solutions. Eur J Biomed Inform. 2017; 13(1): 09-15.

[6] Blobel B. Interoperable EHR Systems-Challenges, Standards, and Solutions. Eur J Biomed Inform. 2018; 14(2): 10-19.

[7] Heubusch K. Interoperability: What it Means, Why it Matters. J AHIMA. 2006; 77: 26-30.

[8] www.who.int/classifications/icd/en/

[16] oauth.net

[17] wiki.hl7.org/index.php?title=201701_CDS_Hooks

[18] www.healthcare-informatics.com/blogs/david-raths/ interoperability/what-cds-hooks-interview-joshmandel-about-fhir-and-clinical

[19] en.wikipedia.org/wiki/Health_Insurance_Portability_ and_Accountability_Act

[9] www.snomed.org/snomed-ct

[20] www.theagenci.com/gdpr/ 